OPEN ACCESS

UWS Academic Portal

\title{
Energy autonomous eSkin
}

Manjakkal, Libu; García Núñez, Carlos; Dahiya, Ravinder

Published in:

SPIE Defense + Commercial Sensing

DOI:

$10.1117 / 12.2520757$

Published: 09/04/2019

Document Version

Peer reviewed version

Link to publication on the UWS Academic Portal

Citation for published version (APA):

Manjakkal, L., García Núñez, C., \& Dahiya, R. (2019). Energy autonomous eSkin. In SPIE Defense + Commercial Sensing: 2019, Baltimore, Maryland, United States. Micro- and Nanotechnology Sensors, Systems, and Applications XI (Vol. 10982). [109821K] (Proceedings of SPIE; Vol. 10982). Society of Photo-Optical Instrumentation Engineers. https://doi.org/10.1117/12.2520757

\section{General rights}

Copyright and moral rights for the publications made accessible in the UWS Academic Portal are retained by the authors and/or other copyright owners and it is a condition of accessing publications that users recognise and abide by the legal requirements associated with these rights. 
Copyright 2019 Society of Photo-Optical Instrumentation Engineers (SPIE). One print or electronic copy may be made for personal use only. Systematic reproduction and distribution, duplication of any material in this publication for a fee or for commercial purposes, and modification of the contents of the publication are prohibited.

Libu Manjakkal, Carlos Garcia Nunez and Ravinder Dahiya "Energy autonomous eSkin," Proc. SPIE 10982, Micro- and Nanotechnology Sensors, Systems, and Applications XI, 109821K (13 May 2019); doi: 10.1117/12.2520757; https://doi.org/10.1117/12.2520757 


\title{
Energy Autonomous eSkin
}

\author{
Libu Manjakkal ${ }^{1}$, Carlos Garcia Nunez $^{2}$, Ravinder Dahiya ${ }^{1 *}$ \\ ${ }^{1}$ Bendable Electronics and Sensing Technologies (BEST) Group, School of Engineering, \\ University of Glasgow, G12 8QQ, Glasgow, UK \\ ${ }^{2}$ SUPA, Institute of Thin Films, Sensors and Imaging, School of Computing, Engineering and \\ Physical Sciences, University of the West of Scotland, PA12BE Paisley, Scotland, UK \\ * Ravinder.Dahiya@glasgow.ac.uk
}

\begin{abstract}
The energy autonomy is a critical feature that would enable better portability and longer operation times for wearable systems. In the next generation of prosthesis and robotics, the operation of multiple components (from few sensors to millions of electronic devices) distributed along surface of an artificial skin will be a major challenge. In this regard, a compact, light-weight and wearable energy system, consisting of energy generators, energy storage devices and low power electronics, is highly needed. The latest discoveries demonstrated with advanced materials (e.g. nanostructures, thin films, organic materials, etc.) have permitted the development of the lighter and wearable sensors, energy harvesters and energy storage devices. Moreover, new techniques to evade wired connection in robotics/prosthesis by using conformable energy generator and storage systems as well as near-field communication data/energy transmission have opened new technological era. This paper presents the development in the field of self-powered e-skin, particularly focusing on the available energy harvesting technologies, high capacity energy storage devices, and high efficiency and low power sensors. The paper highlights the key challenges, critical design strategies, and most promising materials for the development of an energy-autonomous e-skin for robotics, prosthetics and wearable systems.
\end{abstract}

Keywords: Touch sensor, pH sensor, supercapacitor, solar cell, wearable, self-powered, eSkin, healthcare, robotics

\section{INTRODUCTION}

Wearable energy autonomous technology which comprises systems consisting of energy generators, storage and active/passive components, are highly needed for the next generation of point-of-care devices, robotics, environment/infrastructure monitoring devices, automation, space/military applications, etc. [1-5]. The successful integration of flexible, and high efficient energy generators, and high capacity and light-weight energy storage devices to form a self-powered pack (SPP) has a tremendous applicability, e.g. to supply energy continuously to sensors distributed along the surface of an artificial skin. Majority of the power requirements of an artificial skin, also known as e-skin, or wearable sensors and electronics, are provided by bulky batteries with heating drawbacks and heavy weights, or energy harvesters with low energy conversion efficiencies[1]. Moreover, current SPP technology presents additional issues such as low and discontinuous energy scavenging, poor portability and overall wearability, and low life cycles of the storing devices. In addition to this, one of the continues energy generators, photovoltaics produces huge amount of energy (for example solid state flexible photovoltaics have power density $276 \mathrm{w}$. $\mathrm{m}^{-2}$ with $27.6 \%$ of power conversion efficiency [6]) and in which a small portion of energy is only required to operate a low power $\left(\mathrm{nWcm}^{-2}\right)$ sensors. Because of the lack of suitable solutions for energy storage, most of the generated energy is wasted. Significant efforts have been devoted during the last decades to develop flexible energy storage systems capable to carry out a rapid store energy. Among all, supercapacitors (SCs) have highlighted over other wearable and light weight technologies [7-9]. The main reasons is because of SCs can be flexible [8] hold large amount of charge (energy density of depends on type of material $[9,10]$ ) and present quick charging (less than $1 \mathrm{~min}$ ). For these reasons, SCs are particularly promising for motors/actuators utilized in robotics and automation [11]. The ultra-flexibility or bendability properties of SCs with long lifetime ( $>10^{6}$ charging discharging life cycles), environmental friendliness and quick charging (less than $1 \mathrm{~min}$ ), make SCs to have a great integrability with existing wearable energy generators [8] and sensors [5, 12, 13], being a crucial pillar for the development of the next generation of SPPs [7, 14].

Various energy sources such as light, mechanical, thermal and chemical have found potential applications in e-skin [5, 15-17]. Some of the energy generators used to harvest above energy sources have been successfully integrated with suitable storage system resulting in for self-powered applications $[8,14,18]$. The great progress achieved in the field of energy autonomous systems has been summarized in our previous work [1]. From this investigation, we observed that for a fully autonomous e-skin (or wearable system) i.e. working continuously for $24 \mathrm{~h}$ with high stability and 
reliability, a technology with a high power conversion efficiency developed on flexible substrates is highly demanded. Focusing on these aims, we developed (i) flexible energy autonomous transparent tactile skin for robotics/prosthetics [19] (ii) fully flexible self-powered sensors for healthcare monitoring [8] and (iii) solar powered prosthetic hand by integration of flexible SCs and solar cells [7]. Considering multiple sensors (pressure, temperature, chemical, electrochemical, etc.) and its electronic components which are distributed either along the same surface or stacked, the e-skin could bestow robots/prostheses and human different sensing. Among these works, the energy autonomous tactile skin is attractive for the suitability of robots to carry out delicate tasks, e.g. taking care of elderly, high precision operations in space expeditions, rescue actions in catastrophe zones, remote surgery, etc. The autonomous tactile sensors provide very high sensitivity at a very low-pressure range. The developed e-skin can also act as a 'second skin' in humans and can be applied measuring various body parameters such as blood pressure, body temperature, heartbeat etc. Hence, it has potential applications in the field of medical care for health monitoring (instant diagnosis) and also the possibility to coat on a surgery tool to allow the surgeon to get a complete feedback from the patient tissue during the operation [19]. In the second fully self-powered device, we integrated the solar cell and SCs with pH sensors which can be utilized for sweat monitoring applications. The energy stored the SC is used for operating the low powered chemi-resistive sensors. The performance shows that, the self-powered pack has potential to application for online monitoring [8]. We also employed this self-powered pack energy autonomous water quality monitoring sensors [20]. Due to the lack of flexible high energy density storage system, energy autonomy is a major challenge in robotics and prosthetics. The motors/ actuators in fingers required high power to operate (more than $500 \mathrm{~mA}$ of current and above $6 \mathrm{~V}$ of potential). To achieve this this a flexible high energy density SCs which can operate in voltage $2.25 \mathrm{~V}$ was developed. The SCs stacked together and integrated with solar cell to operate the actuators of fingers of the prosthesis [7].

In this paper, we review current state-of-the-art energy autonomous systems, showing our latest work on e-skin for healthcare and robotic applications. In section 2, we discuss the fabrication strategies and processing of flexible tactile/chemical sensors, energy storage systems (SCs) and self-powered system, including the brief description of their integration in SPPs. In section 3, we present our latest results obtained in matter of robotics and medical applications.

\section{EXPERIMENTAL SECTION}

\subsection{Fabrication of touch sensors for e-skin applications}

The fabrication steps of graphene based interdigitated transparent and flexible capacitive touch sensors were reported in our previous work [19]. In this work, the CVD graphene were transferred on the top of ultra flexible poly vinyl chloride (PVC) susbstrate by e-beam evaporation and shadow mask method. A computer controlled blade cutting machine (Silhouette Cameo), is used to shape the single layer graphene as co-planar interdigitated electrodes and various patterns. Finally as protetive/encapsulative layer between external stimuli and graphene, a thick PDMS layer was spin coated on the top of graphene film. Figure 1a shows the schematic and image of the fabricated touch sensor that is eventually integrated on a robotic hand for e-skin application.

\subsection{Fabrication of flexible $\mathrm{pH}$ sensors for healthcare}

A flexible chemi-resistive $\mathrm{pH}$ sensor was fabricated on flexible polyethylene glycol terephthalate (PET) substrate as shown in Figure $1 \mathrm{~b}$ by screen printing method. $\mathrm{CuO}$ nanostructured material was used as sensitive electrode. For this, $\mathrm{CuO}$ nanomaterial was synthesised by hydrothermal method and paste based on this material was prepared. An Ag conductive pads was used as an electrode. The details of material preparation and sensors fabrication was given in previous reports $[8,21]$. The resistance variation of the sensor with varying $\mathrm{pH}$ value of solution was monitored through a multimeter.

\subsection{Fabrication of supercapacitors for energy storage}

For energy storage, we developed two types of SCs (i) Graphene-Ag-3D Graphene Foam based SC (GFSC) and (ii) Graphene-Graphite Polyurethane Composites based SC (GS/GPUSC). In this GFSC electrode structure consists of a highly conductive graphene sheet (GS) as a current collector. A free-standing 3D structured graphene foam (GF) bonded on the top of GS by using Ag conductive epoxy as an adhesive layer. The steps of fabrication of GFSC is described in our previous work. The cross-section of schematic of GFSC is shown in Figure 1c. For GS/GPUSC fabrication we prepared a new graphite-polyurethane (GPU) composite. GPU composite were printed on the top of GS (GS act as a current collector), as shown in Figure 1d. The method of GPU film and GS/GPUSC fabrication reported elsewhere[7]. The electrochemical performances of both SCs were investigated by cyclic voltammetry (CV), electrochemical impedance spectroscopy (EIS) and galvanostatic charging - discharging (GCD) methods. The 
performances under static and dynamic bending and long-life cycle (> 25000 for GFSC and > 15000 for GS/GPUSC) charging-discharging of SCs were investigated.

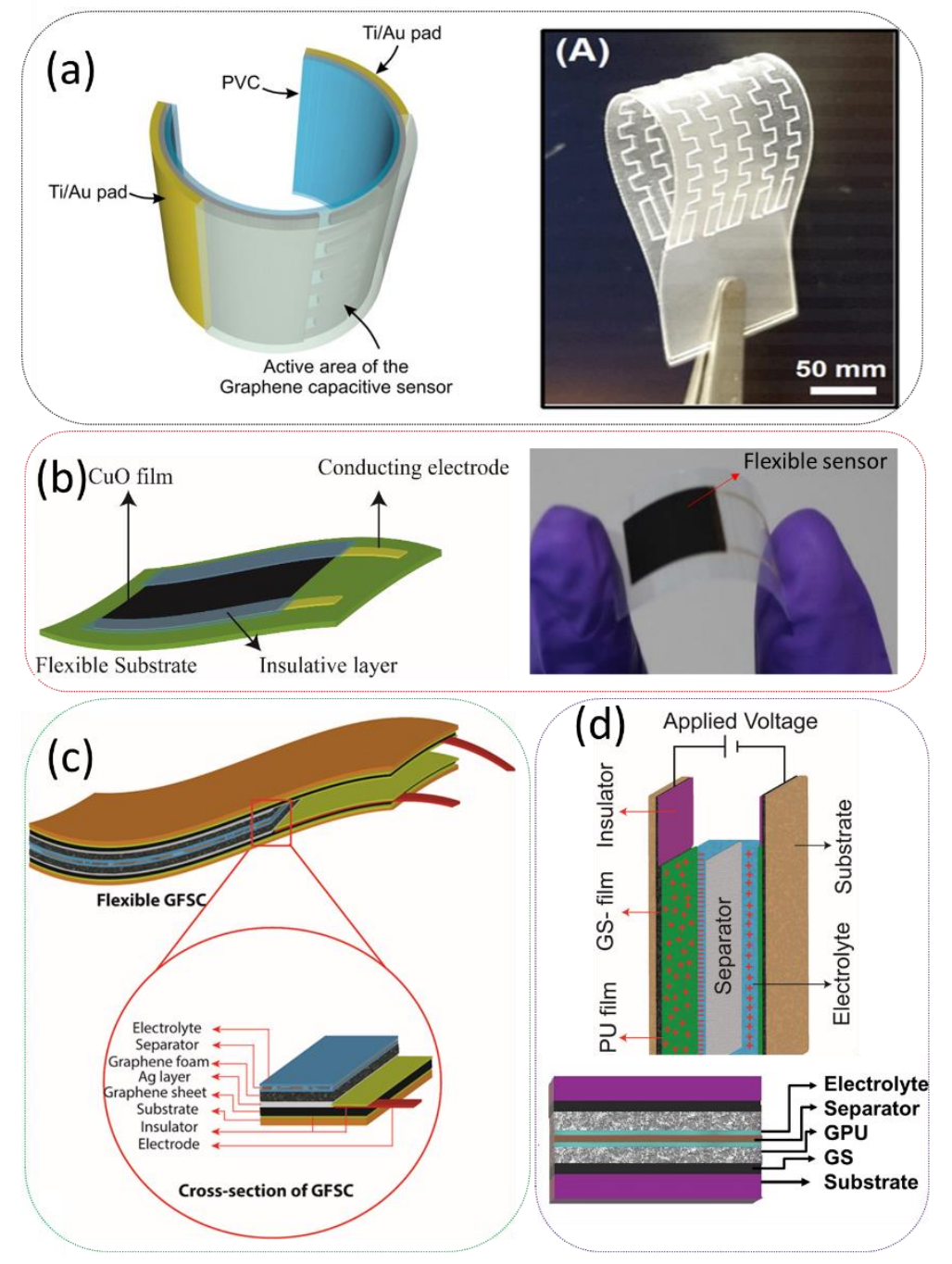

Figure 1: (a) Schematic representation and image of flexible tactile e-skin. Reprinted with permission from García Núñez et al.[19] Copyright (C) 2017, John Wiley and Sons. (b) Schematic and image of flexible pH sensor [8, 21] Reprinted with permission from Elsevier [21]. (c) Flexible graphene foam- based SC (GFSC) with cross-sectional view. Published in [8]. (d) Layered electrode-based GS/GPUSC with electrode layers [7].

\subsection{Development of energy autonomous e-skin for robotics/prosthetics}

To validate the graphene touch sensors for e-skin, the sensors were integrated at the intermediate and proximal phalanges of i-Limb, a state-of-the-art bionic hand (Figure 2a). Due to the different size of the phalanges, sensors placed at intermediate phalanges have less active area than those placed at proximal phalanges. Figure 2a shows a magnified image of sensors on each phalange, with clearly visible interdigitated electrodes. The response of graphene sensors was converted from capacitive variation to a voltage through a readout interface circuitry which was designed and implemented in a flexible polyimide substrate. The printed circuit had option to read ten sensors from the intermediate and proximal phalanges of five fingers of i-Limb. Transparency of all the layers used in our touch sensor, including the protective layer (PDMS), the capacitive layer (graphene), and the flexible substrate (PVC), was used by integrating the graphene touch sensors directly on top of solar cells [19].

\subsection{Development of fully flexible self-powered pack (FSPP) for healthcare monitoring}

For the fabrication of the FSPP, flexible GFSC modules are connected (placed underneath) to a flexible amorphous silicon photovoltaic cell. The obtained FSPP is integrated to the flexible chemi-resistive pH sensor. Schematic of FSPP with sensor is shown in Figure $2 b$. The energy pack such as FSPP act as a voltage source of the sensor. For the power management, the energy harvested by the solar cell was integrated to the GFSC through an evaluation board SPV1050 ULP (from STMicroelectronics). For sensing application, the variation of resistance of $\mathrm{CuO}$ electrodes in different $\mathrm{pH}$ 
value of solutions were tested. Dilute $\mathrm{HCl}$ or $\mathrm{KOH}$ was added into distilled water to vary the $\mathrm{pH}$ value of solution. The performances FSPP were investigated under above dynamic cyclic bending conditions and 1 sun and 0.5 sun conditions [8].

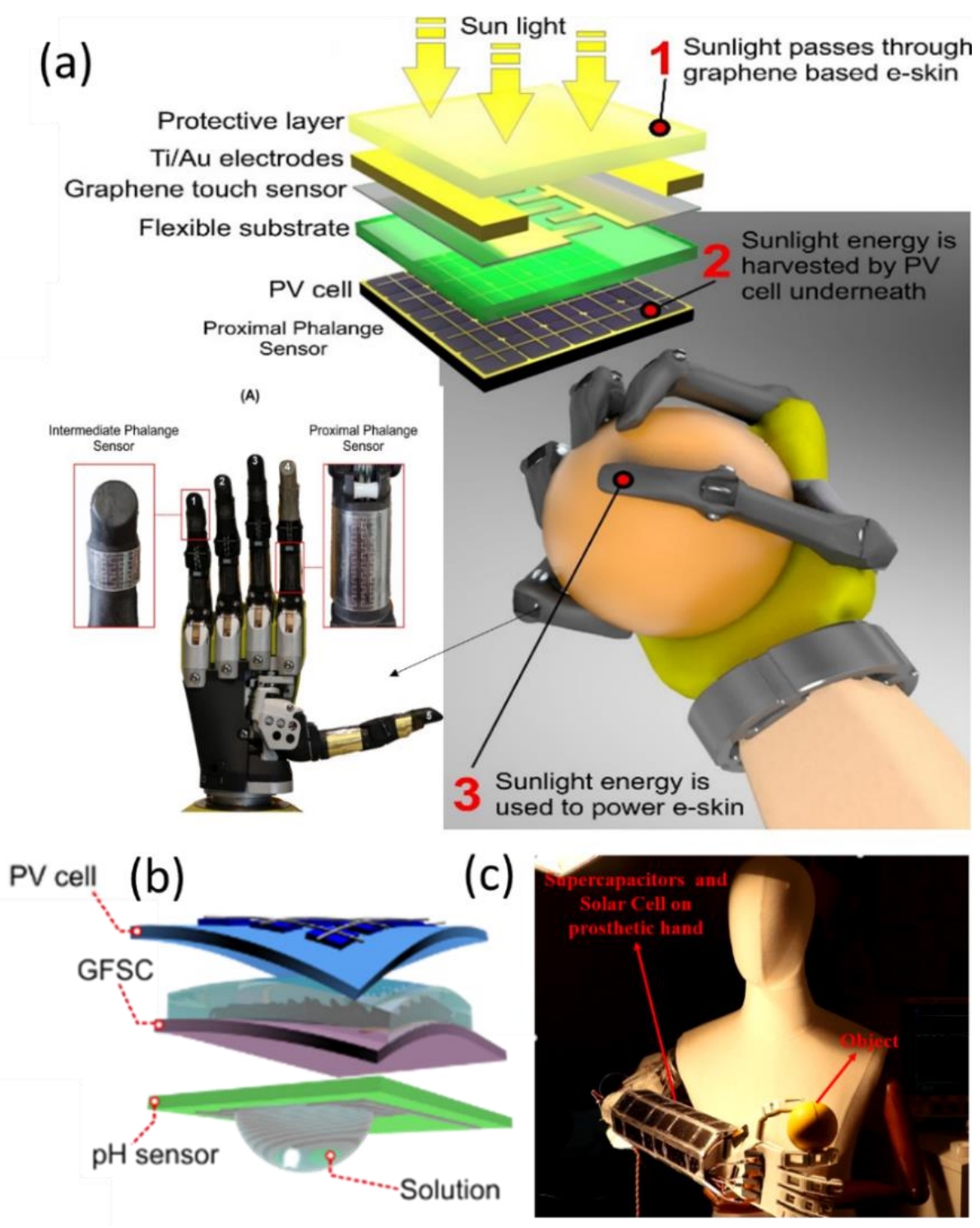

Figure 2: (a) Schematic representation and image of flexible tactile e-skin on robotic hand along with integration of solar cell [19] (b) Schematic representation of stacking of solar cell, GFSC and pH sensor [8]. (c) Image of SC and solar cell integrated on prosthetic hand [8].

\subsection{Development of solar powered prosthetic/robotic hand}

Energy autonomy is a major challenge in robotics and prosthetics. The developed GS/GPUSCs integrated with solar cell have also been used as an energy storage device in prosthetic/robotic hand. For the demonstration, the finger actuators of the hand are powered by the PV cells and SCs (Figure 2c). The electrical schematic and block diagram of the system are discussed in our previous report. In this prosthetic/ robotic hand the power consumption of the actuators varies from $50 \mathrm{~mA}$ (under no load), $250 \mathrm{~mA}$ (at $50 \mathrm{~N}$ load) to maximum current of $550 \mathrm{~mA}$ when stalled. A series array of GS/GPSCs is wrapped around the forearm of the hand. Under bending conditions, when the light illuminated on the curved surface of the solar it generates voltage around $7.6 \mathrm{~V}$ and this generated voltage stored the SCs. After the SC gets charged, through programmed circuit, the voltage is supplied to the motors and hence the index and thumb fingers are perform to grab and release object [7]. 


\section{RESULTS AND DISCUSSION}

\subsection{Energy Autonomous e-skin for robotics}

After the fabrication of graphene based flexible capacitive touch sensors, sensor response has been studied under quasistatic touching conditions. Using a LCR meter (Keysight E4980AL), base capacitance $\left(C_{0}\right)$ of sensors was measured in air ambient conditions and typically obtained values in the range of $\sim 8-9 \mathrm{pF}$. $C_{0}$ was also measured before the deposition of PDMS protection layer, showing a value of $5.5 \mathrm{pF}$. This shows that the PDMS protective layer increases the sensor capacitance, which is largely due to the higher dielectric constant of PDMS with respect to the air $\left(\varepsilon=3 \varepsilon_{0}\right.$, $\varepsilon_{0}$ being the dielectric constant of the air). It is worth noting that the thickness of PDMS strongly influences the $C_{0}$, i.e. thicker the PDMS, lower is the $C_{0}[19]$.

In order to analyse thoroughly the tactile response of sensors, quasi-static touching experiments were carried out by using a linear stage motor to exert periodic pulses of pressure on the device active area Figure 3a. Figure $3 \mathrm{~b}$ represents the capacitive response $\left(\Delta C / C_{0}\right)$ measured over time on graphene based flexible capacitive touch sensors. The high accuracy of the linear stage motor enables us to move the PDMS rubber down to micrometric distances and allows to exert periodic pressures of different magnitudes on the sensors. In this regard, external pressures applied on the sensors sensitive area overtime, and simultaneously recorded the capacitance change with the LCR meter Figure $3 \mathrm{~b}$. All sensors based on interdigitated electrodes with different geometries including lines and meandered shapes were analyzed (Figure 1a). Among various geometries, the meandered shaped interdigitated electrodes provided one of the highest capacitance modulation and uniform response along with the scanned pressure range. Following these findings, we further analysed the response of this specific sensor sample. This capacitive sensor shows stable response for all the analysed pressures, i.e. pressures of $9.8,26.7,47.3,64.6$ and $72.4 \mathrm{kPa}$ resulting in $\Delta C / C_{0} 1.9 \%, 10.5 \%, 17.3 \%$, $25.5 \%$, and $53.1 \%$, respectively, and that was independently on the measurement routine, i.e. from low to high pressures or vice versa; this result can be explained by arguing that the thin thickness of the PDMS reduces stretching/releasing process period during and after the pressure is applied on the sensor. More importantly, $\Delta C / C_{0}$ presents different values depending on the applied pressure. This pressure sensitivity is a novel and interesting behavior in co-planar based structure, especially because the conventional co-planar or staggered structures (co-planar capacitor type I in Figure 3c) commonly used in commercial capacitive touch screens can only sense presence or absence of touch. Other common approach reported for graphene capacitive sensors, is the use of graphene as two conductive parallel electrodes separated with a stretchable material (parallel capacitor in Figure 3c). In this approach, the graphene directly experiences the pressure, which may damage the graphene and may lead to reliability issues. The co-planar structure presented here (type II in Figure 3c), shows an additional feature compared to type I, i.e. the sensitivity to the pressure. The origin of their pressure sensitivity is mainly attributed to the change of the PDMS dielectric constant under compression. The cured PDMS has porous structure, the volumetric density of voids in PDMS decreases (Figure $3 \mathrm{c})$ when it is pressed. This leads to a change in its effective dielectric constant and thus the measured capacitance.

The sensitivity of the sensor was calculated by $S=\delta\left(\Delta C / C_{0}\right) / \delta P$, where $P$ is the applied pressure. $S$ depends on the applied pressure range, as clearly observed in the inset of Figure $3 \mathrm{~b}$. From 0 to $20 \mathrm{kPa}$, sensors present a sensitivity of $9.3 \times 10^{-3} \mathrm{kPa}^{-1}$, between 20 and $60 \mathrm{kPa}$ they show $4.3 \times 10^{-3} \mathrm{kPa}^{-1}$, and at pressures higher than $60 \mathrm{kPa}$ sensors present a sensitivity of $7.7 \times 10^{-3} \mathrm{kPa}^{-1}$. Capacitive sensors fabricated in this work present $S$ lower than those obtained in capacitive sensors based on conductive porous sponges that show up to $0.26 \mathrm{kPa}^{-1}$ in the range of pressure below 2 $\mathrm{kPa}$. In contrast, our sensors show similar sensitivities along with a wider range of pressures up to $80 \mathrm{kPa}$, and have attractive properties such as transparency, thin structure, and sensitivity to the pressure, which are all promising features for $e$-skin applications. In addition, the sensor developed in this work shows a unique behavior that has not been observed before, which is the second increase of the sensitivity above $60 \mathrm{kPa}$. That behavior makes this device even more attractive in a broad range of pressures, where other reported pressure sensors show loss of sensitivity with pressure [19].

In order to evaluate the functionality of the graphene capacitive touch sensors, especially when they are integrated non-planar surfaces, the measured response of sensors is compared on both flat (Figure 3d) and non-planar surfaces (Figure 3e). Quasi-static measurements of $\Delta C / C_{0}$ were carried out in flat and bending mode, using flat and bent PDMS soft probes to touch the same active area in both scenarios. Figure $3 \mathrm{f}$ shows that the response, i.e. $\Delta C / C_{0}$, of the sensors remains unaffected in both cases. This is mainly because of the intrinsic mechanical robustness of graphene, which preserves electrical properties after transfer to either flat or non-planar surfaces. In addition, the good conformal contact formed between graphene and PVC substrate during the hot lamination transfer procedure making the device architecture more robust and very stable even under the stresses experienced during bending.

Due to the intrinsic transparency of all layers existing in the sensor, incident light is expected to be efficiently transmitted through the whole structure reaching the surface of the solar cell. Initially, we analyzed the optical transmission of the sensors. The transmittance (T) and reflectance (R) measurements of $125 \mu \mathrm{m}$ thick PVC as a reference substrate and graphene on PVC is shown in Figure 3g. Figure $3 \mathrm{~h}$ shows the current-voltage (I-V) 
characteristics of this solar cell were measured after integration of different layers atop of its surface, including a graphene on PVC, patterned graphene on PVC, and patterned graphene on PVC encapsulated with PDMS. From the measurement of the pen circuit voltage $\left(\mathrm{V}_{\mathrm{oc}}\right)$ and short circuit current $\left(\mathrm{I}_{\mathrm{sc}}\right)$ (shown in Figure $\left.3 \mathrm{~h}\right)$, we found that both $\mathrm{V}_{\mathrm{oc}}$ and $\mathrm{I}_{\mathrm{sc}}$ parameters decrease as we add a layer on top of the solar cell surface. This observation reveals that graphene touch sensor is absorbing/reflecting partially the incident light and some light may get scattered as well within the graphene touch sensor and interface before reaching the solar cell. From the power-voltage characteristics of the solar cell (shown in Figure 3i), we deduce a maximum power of around $1.48 \mathrm{~mW}$ with a maximum voltage of $1.55 \mathrm{~V}$ and maximum current of $-0.95 \mathrm{~mA}$. Here we found that the solar cell used in this work can produce a power of $160 \mu \mathrm{W}$. $\mathrm{cm}^{-2}$. If the tactile skin presented here were to cover the glabrous skin of a human hand (average area around $120 \mathrm{~cm}^{2}$ ), the solar cells used in this work will generate $19.2 \mathrm{~mW}$, which is more than the power needed to drive an $e$-skin module, i.e. a graphene touch sensor. Further advancements in terms of high efficiency energy harvesting, energy storing and together, less implementation could lead to a full autonomy of the $e$-skin. The effective integration of both technologies could allow in future the charging of batteries either to power actuators or to power up integrated circuits (ICs) on large area $e$-skin, leading to self-powered robotic/prosthetic limbs with tactile sensitivity [19].

(a)

(c)
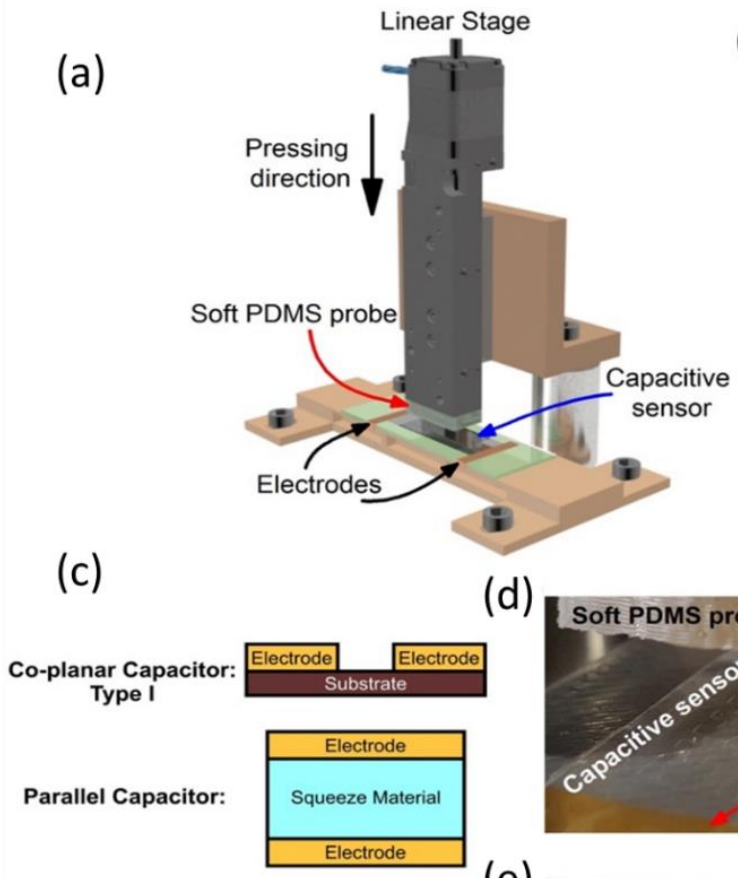

Co-planar Capacitor: Type ॥

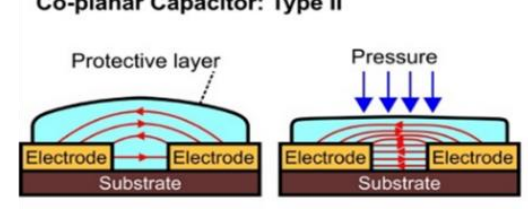

(d)

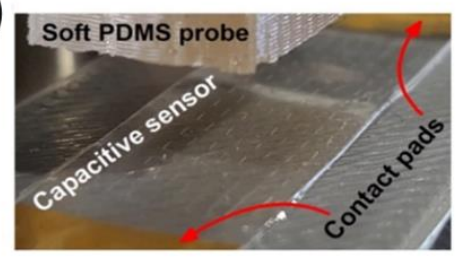

(e)
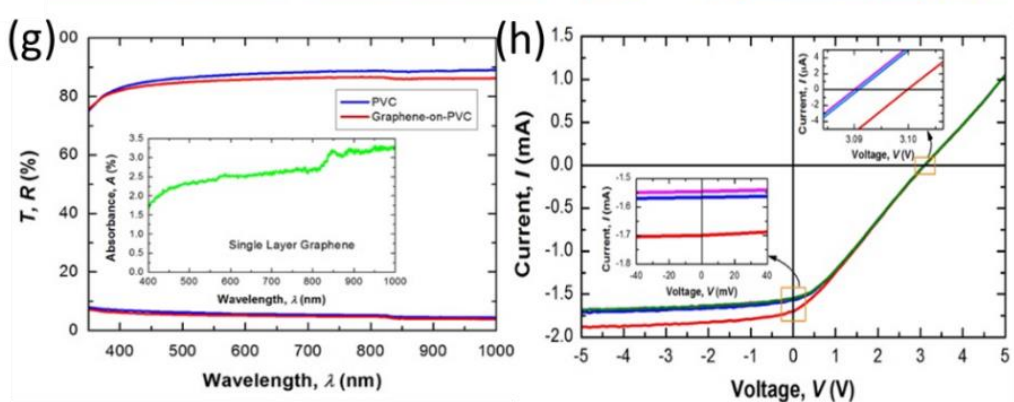

(b)

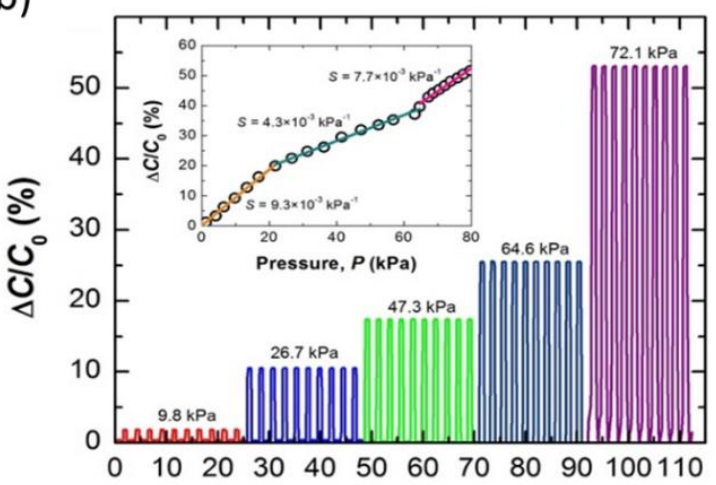

(f)

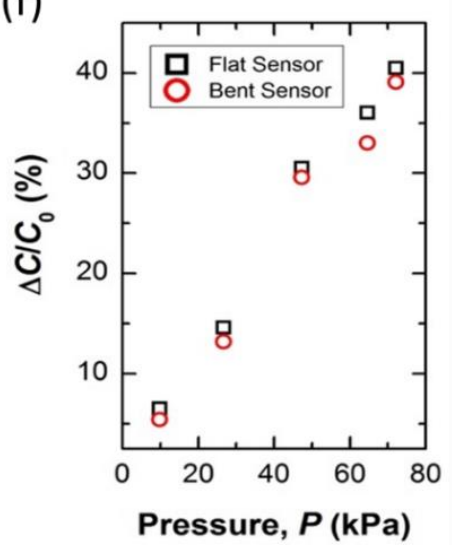

Figure 3. Capacitive response of graphene based flexible sensors. (a) 3D schematic illustration of experimental setup. (b) Response of the graphene based flexible sensor under the quasi-static application of pressure overtime for various pressures; inset: extracted sensitivities for wide pressure regime. (b) Co-planar and parallel based capacitors; type I capacitor is sensitive to touch and proximity, whereas type II and parallel capacitors are sensitive to pressure. (d,e) Photographs of capacitive sensors under test in flat and bending conditions. (f) $\Delta C / C_{0}$ vs pressure measured at flat (f) 
and bending (e) conditions. (g) Transmittance and reflectance spectra of single-layer graphene-on-PVC and a PVC reference substrate; inset: absorbance of single-layer graphene (h) I-V and (i) $\mathrm{P}-\mathrm{V}$ characteristics of the solar cell after integration of samples consisting of PVC, graphene-on-PVC, and graphene-on-PVC with a PDMS protective layer. Reprinted with permission from García Núñez et al.[19] Copyright @ 2017, John Wiley and Sons.

\subsection{Energy autonomous sensors for healthcare}

Prior to implement the FSPP for sensors, we initially measured the storage ability of GFSC. The charging discharging curve in Figure 4a show that, the fabricated the porous GFSC have a specific capacitance of $38.25 \mathrm{mF} \mathrm{cm}^{-2}$ for current

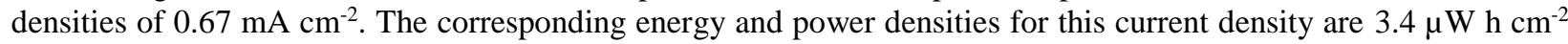
and $0.27 \mathrm{~mW} \mathrm{~cm}^{-2}$ respectively. The variation of capacitance and energy density with current density is shown in Figure 4b. After testing the GFSC, the fabricated FSPP have been evaluated under 0.5 sun and 1 sun conditions. This is similar to the observation during a cloudy and sunny day. Figure $4 \mathrm{c}$ shows the charging discharging curves for different light intensities. The measured charging- discharging times and potentials for these light intensities are summarized Table in the inset of Figure 4c. From this measurement, it was found that, the FSPP is suitable for the wearable devices which required low operating power. This FSPP has been used as a power source, to power continuously a wearable $\mathrm{pH}$ sensor. To power a wearable $\mathrm{pH}$ sensor, the GFSC in the FSPP is fully charged by suing the solar cell under 1 sun condition. A voltage divider used to limit the charging current $(0.5 \mathrm{~mA})$ and voltage $(0.8 \mathrm{~V})$. The sensor required a voltage of $<250 \mathrm{mV}$ and is supplied form this FSPP. The low power consumption of the $\mathrm{pH}$ sensor (range of $\mathrm{nW} \mathrm{cm}$ ) shows that the connection of the GFSC with a power density in the range of $\mathrm{mWcm}^{-2}$ is suitable as a power supply for the sensor. Moreover, very fast response, with a response time of less than $5 \mathrm{~s}$ from $\mathrm{pH}$ 6.38 to $\mathrm{pH} 4$ and nearly $12 \mathrm{~s}$ from $\mathrm{pH} 5$ to $\mathrm{pH} 5.8$ confirms the advantage of developed self-powered system for wearable application. The measured resistance variation of flexible $\mathrm{pH}$ sensor as a function of different $\mathrm{pH}$ value of solutions is shown in Figure 4d. For continuous online monitoring application, the performance of pH sensor was characterized with and without sunlight in the FSPP. The analysis shows that, this FSPP with a wearable sensor platform can be further extended towards a $24 \mathrm{~h}$ self-powering systems. Hence, if there is no sunlight, the GFSC can be used to power sensors [8].
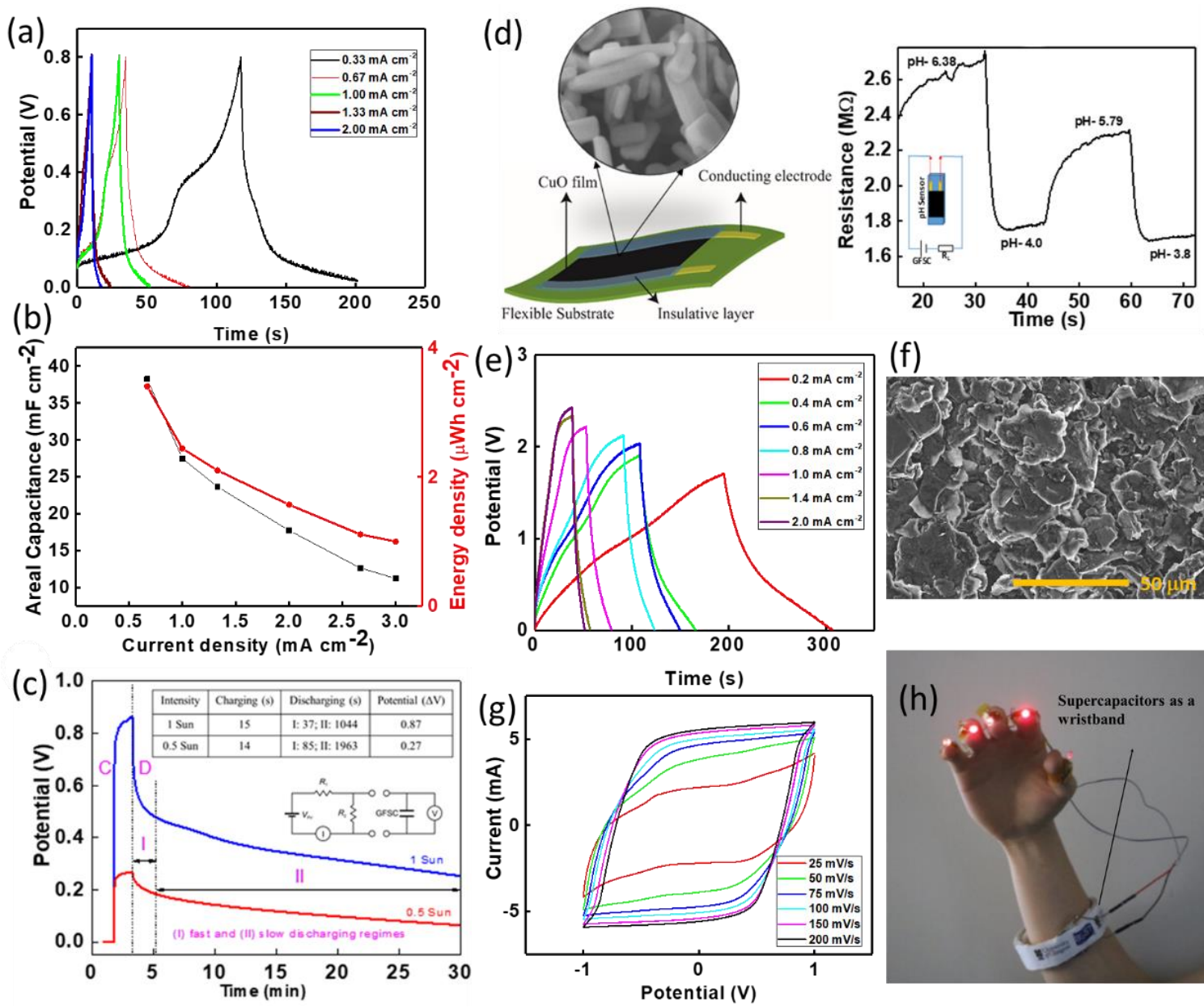

(f)
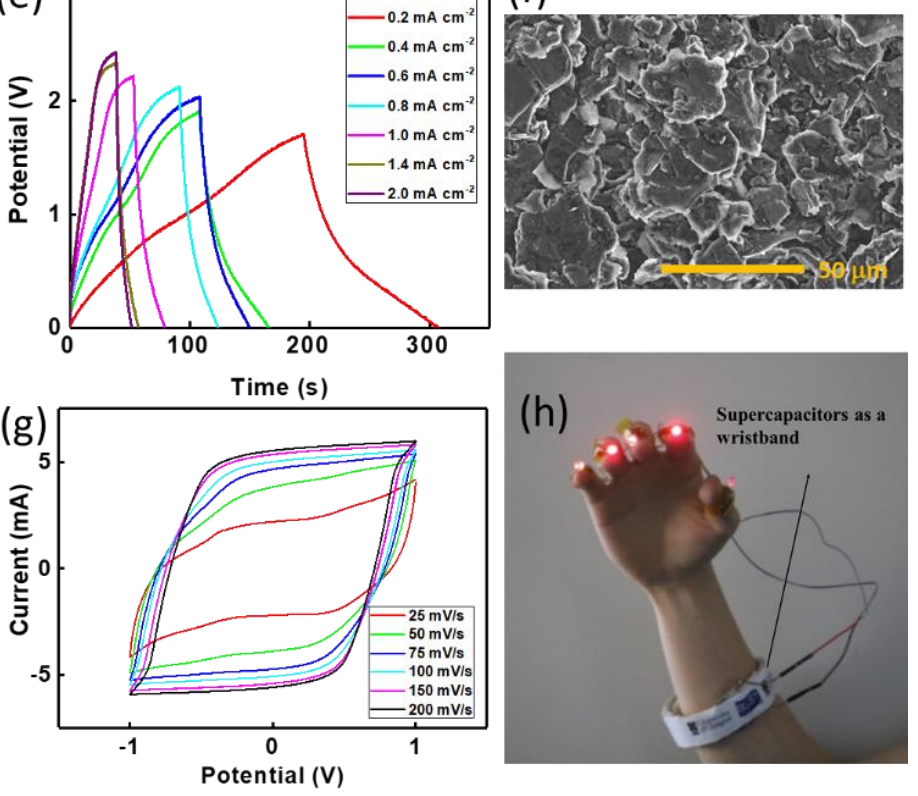
Figure: 4 (a) Charging-discharging curve for GFSC. (b) The variation of capacitance and energy density with current density for GFSC. (c) The charging discharging curve of FSPP with different light intensity. (d) Image of sensor (surface morphology of the sensitive electrode) and the variation of resistance of the sensor [8]. (e) Charging discharging of GS/GPUSC under voltage saturation (f) the surface morphology of GPU film (g) CV analysis under different scan rate for GS/GPUSC [7] and (h) Performance of GS/GPUSC based wristband for wearable application [7].

Further advances in the energy autonomous systems in wearable applications, such as to power multiple nano-/microdevices, e-skin components, prosthetics, transmitting the sensor data to smartphone, etc., a high-power density energy pack is required. For high energy and power density application, the energy storage device should be working on high operating voltage. The fabricated flexible GS/GPUSC shows an operating voltage of $2.25 \mathrm{~V}$ (charging-discharging curve is shown in Figure 4e) and high storage capacity in high current densities [7]. The high electrically conductive $\left(0.318 \mathrm{~S} \mathrm{~cm}^{-1}\right)$ GPU composite based SCs have maximum energy and power densities of $10.22 \mu \mathrm{Wh} \mathrm{cm}^{-2}$ and 11.15 $\mathrm{mW} \mathrm{cm}$, at a current density of $10 \mathrm{~mA} \mathrm{~cm}$. The significant gain in the electrochemical and supercapacitive performance of GS/GPUSC is due to the nanostructured and rough surface (Figure 4f )of GPU exhibit excellent electroactive surface for the electrolyte (CV curve for different scan rate is shown in Figure $4 \mathrm{~g}$. The low ionic diffusion resistance and wettability (low hydrophobicity) leads to high conductance for ionic/ electronic exchange of the electrode. The wearable application of the SC was proved by developing a flexible wristband, as shown in Figure 4h. The charging discharging performance of this wristband GS/GPUSC shows that, this flexible SC can be used for high power density applications. The high energy and power density of the developed SCs have also been used as an energy storage device to operate fingers of prosthetic hand. For this the GS/GPUSC was integrated to solar cell. After the GS/GPUSC gets charged, a switch (SW) is toggled to turn on the prosthesis. This results in the GS/GPUSC powering all the micro servo motors. A diode 1N5404 is used to lower the supply voltage to suit the 1688 microcontroller's preferred operating voltage $(4.0 \mathrm{~V}-5.5 \mathrm{~V})$. The microcontroller is programmed to output pulse width modulated (PWM) signal to control the position of the various servos on various pins according to requirement. The index and thumb fingers are actuated through PWM to perform grab and release action. This energy autonomous system offers an attractive solution for future energy autonomous prosthetics [7] and can be extended to other application areas of wearable systems [20-27].

\section{CONCLUSION}

In this paper, we discussed the development of energy autonomous eSkin systems for robotics and healthcare applications. The integration of fabricated sensors (tactile sensor and $\mathrm{pH}$ sensor) and energy storage systems (SCs) and self-powered system is presented. This presented concept is a step forward towards a new generation of energy autonomous tactile skin by harvesting ambient light energy to power up sensor-transducer ICs or the robotic/prostheic hand. This approach can be further exploited by integrating our flexible touch sensors on flexible and stretchable solar cells, enabling a new concept of energy autonomous robotics and prosthesis.

\section{ACKNOWLEDGEMENT}

This work was supported by the EPSRC Engineering Fellowship for Growth - PRINTSKIN (EP/M002527/1) and neuPRINTSKIN (EP/R029644/1).

\section{REFERENCES}

[1] C. García Núñez, L. Manjakkal, R. Dahiya, "Energy autonomous electronic skin", npj Flexible Electronics 3(1), 1 (2019).

[2] W. Dang, L. Manjakkal, W.T. Navaraj, L. Lorenzelli, V. Vinciguerra, R. Dahiya, "Stretchable wireless system for sweat pH monitoring", Biosens. Bioelectron. 107, 192-202 (2018).

[3] R.S. Dahiya, G. Metta, M. Valle, G. Sandini, "Tactile Sensing-From Humans to Humanoids", IEEE T. Robot. 26(1), 1-20 (2010).

[4] R. Dahiya, E-Skin: "From Humanoids to Humans", Proc. IEEE 107(2), 247-252 (2019).

[5] X. Pu, M. Liu, X. Chen, J. Sun, C. Du, Y. Zhang, J. Zhai, W. Hu, Z.L. Wang, "Ultrastretchable, transparent triboelectric nanogenerator as electronic skin for biomechanical energy harvesting and tactile sensing", Sci. Adv. 3(5), 1700015 (2017). 
[6] B.M. Kayes, H. Nie, R. Twist, S.G. Spruytte, F. Reinhardt, I.C. Kizilyalli, G.S. Higashi, "27.6\% Conversion efficiency, a new record for single-junction solar cells under 1 sun illumination", 2011 37th IEEE Photovoltaic Specialists Conference, 000004-000008 (2011).

[7] L. Manjakkal, W.T. Navaraj, C.G. Núñez, R. Dahiya, "Graphene-Graphite Polyurethane Composite Based HighEnergy Density Flexible Supercapacitors", Adv. Sci. 6(7), 1802251 (2019).

[8] L. Manjakkal, C.G. Núñez, W. Dang, R. Dahiya, "Flexible self-charging supercapacitor based on graphene-Ag-3D graphene foam electrodes", Nano Energy 51, 604-612 (2018).

[9] Z. Lin, E. Goikolea, A. Balducci, K. Naoi, P.L. Taberna, M. Salanne, G. Yushin, P. Simon, "Materials for supercapacitors: When Li-ion battery power is not enough", Mater. Today 21(4), 419-436 (2018).

[10] A. Borenstein, O. Hanna, R. Attias, S. Luski, T. Brousse, D. Aurbach, "Carbon-based composite materials for supercapacitor electrodes: a review", J. Mater.Chem. A 5(25), 12653-12672 (2017).

[11] M. Horn, J. MacLeod, M. Liu, J. Webb, N. Motta, "Supercapacitors: A new source of power for electric cars?", Economic Analysis and Policy 61, 93-103 (2019).

[12] N. Yogeswaran, W.T. Navaraj, S. Gupta, F. Liu, V. Vinciguerra, L. Lorenzelli, R. Dahiya, "Piezoelectric graphene field effect transistor pressure sensors for tactile sensing", Appl. Phys. Lett. 113(1), 014102 (2018).

[13] L. Manjakkal, D. Shakthivel, R. Dahiya, "Flexible Printed Reference Electrodes for Electrochemical Applications", Adv. Mater. Technol. 3(12), 1800252 (2018).

[14] J. Luo, F.R. Fan, T. Jiang, Z. Wang, W. Tang, C. Zhang, M. Liu, G. Cao, Z.L. Wang, "Integration of microsupercapacitors with triboelectric nanogenerators for a flexible self-charging power unit", Nano Research 8(12), 39343943 (2015).

[15] A.J. Bandodkar, J.-M. You, N.-H. Kim, Y. Gu, R. Kumar, A.M.V. Mohan, J. Kurniawan, S. Imani, T. Nakagawa, B. Parish, M. Parthasarathy, P.P. Mercier, S. Xu, J. Wang, "Soft, stretchable, high power density electronic skin-based biofuel cells for scavenging energy from human sweat", Energy Environ. Sci. 10(7), 1581-1589 (2017).

[16] S. Park, H. Kim, M. Vosgueritchian, S. Cheon, H. Kim, J.H. Koo, T.R. Kim, S. Lee, G. Schwartz, H. Chang, Z. Bao, "Stretchable Energy-Harvesting Tactile Electronic Skin Capable of Differentiating Multiple Mechanical Stimuli Modes", Adv. Mater. 26(43), 7324-7332 (2014).

[17] W. Taube Navaraj, C. García Núñez, D. Shakthivel, V. Vinciguerra, F. Labeau, D.H. Gregory, R. Dahiya, "Nanowire FET Based Neural Element for Robotic Tactile Sensing Skin", Frontiers in Neurosci. 11(501) (2017).

[18] T. An, Y. Ling, S. Gong, B. Zhu, Y. Zhao, D. Dong, L.W. Yap, Y. Wang, W. Cheng, "A Wearable Second SkinLike Multifunctional Supercapacitor with Vertical Gold Nanowires and Electrochromic Polyaniline", Adv.Mater. Technol. 4(3), 1800473 (2019).

[19] C.G. Núñez, W.T. Navaraj, E.O. Polat, R. Dahiya, "Energy-Autonomous, Flexible, and Transparent Tactile Skin", Adv. Funct. Mater. 27(18), 1606287 (2017).

[20] L. Manjakkal, F. Nikbakhtnasrabadi, R. Dahiya, "Energy Autonomous Sensors for Water Quality Monitoring", IEEE SENSORS, 1-4 (2018).

[21] L. Manjakkal, B. Sakthivel, N. Gopalakrishnan, R. Dahiya, "Printed flexible electrochemical pH sensors based on CuO nanorods", Sens.Actuat. B: Chem.263, 50-58 (2018).

[22] L. Manjakkal, W. Dang, N. Yogeswaran, R. Dahiya, "Textile Based Potentiometric Electrochemical pH Sensor for Wearable Applications", Biosensors, Vol 9 (14), 1-12 (2019).

[23] C. G. Nunez, F. Liu, W. Taube, A. Christou, D. Sakthivel, R. Dahiya, "Heterogeneous Integration of Contactprinted Semiconductor Nanowires for High Performance Devices on Large Areas", Microsystems and Nanoengineering, 4 (2018).

[24] S. Gupta, W. T. Navaraj, L. Lorenzelli, R. Dahiya, "Ultra-Thin Chips for High-Performance Flexible Electronics", NPJ Flexible Electronics, 2 (2018).

[25] R. Dahiya, W. T. Navaraj, S. Khan, E. O. Polat, "Developing Electronic Skin with Sense of Touch", Information Display, Vol. 31 (4), 6-10 (2015).

[26] W. Taube, S. Gupta, L. Lorenzelli, R. Dahiya, "Wafer Scale Transfer of Ultra-Thin Silicon Chips on Flexible Substrates for High Performance Bendable Systems", Adv. Electron. Mater, 1700277 (2018).

[27] C. G. Nunez, A. Vilouras, W. T. Navaraj, F. Liu, R. Dahiya, "ZnO Nanowires based Flexible UV Photodetector System for Wearable Dosimetry”, IEEE Sens. J., 18 (19), 7881-7888 (2018). 